\title{
Pregnancy Following Bariatric Surgery-Medical Complications and Management
}

\author{
Radwan Kassir $^{1,2} \cdot$ Marie-Pierre Goiset $^{1}$ - Olivier Tiffet ${ }^{1}$
}

Published online: 29 August 2016

(C) Springer Science+Business Media New York 2016

Keywords Bariatric surgery $\cdot$ Anemia $\cdot$ Obesity .

Postabsorptive hypoglycemia $\cdot$ Pregnancy

In the adult obese population, there is a large percentage of women of childbearing age. It means a higher rate of pathological pregnancies due to an increased risk of developing gestational diabetes mellitus (GDM), gestational hypertensive disorders, preeclampsia, and fetal macrosomia.

Narayanan RP et al. concluded that nutrient deficiencies may vary depending on the type of surgery; it is important to optimize the nutritional status of women prior to and during pregnancy. Dietary management should include adequate protein and calorie intake and supplementation of vitamins and micronutrients [1].

In the same time, we do not agree with Narayanan RP et al., and we agree that the pregnant women with history of bariatric surgery were significantly more anemic than those with no history of surgery and with the three types of surgery that is to say biliopancreatic diversion (BPD), Roux-en-Y gastric bypass (RYGB), and sleeve gastrectomy (SG).

In the study of N. Mead et al., hemoglobin and hematocrit levels decreased significantly during pregnancy in all three groups corresponding to the different types of surgery, but none received intravenous iron administration [2]. Vitamin B12 levels decreased after all types of surgery but no significant further decrease during pregnancy, contrary to folic acid levels which

Radwan Kassir

Radwankassir42@hotmail.Fr

1 Department of General Surgery, CHU Hospital, Jean Monnet University, Saint Etienne, France

2 Department of Bariatric Surgery, CHU Hospital, Jean Monnet University, Avenue Albert Raimond, 42270 Saint Etienne, France

increased both after surgery and during pregnancy (probably due to supplementation guidelines). Hypoproteinemia was seen only after BPD. There was a significant improvement in the metabolic profile of all women who underwent surgery regarding blood glucose levels.

There are good pregnancy outcomes after all types of bariatric surgery provided nutritional supplement guidelines are followed. However, closer monitoring is required after malabsorptive procedures especially regarding protein nutrition, as hypoproteinemia may have an effect on the incidence of LBW and SGA.

Vitamin B12 levels should be monitored closely after all types of bariatric surgery, especially during pregnancy. Investigation on deficiencies of other nutrients, including vitamin D, and possible associations with neonatal outcomes would also be of great interest.

Moreover, bariatric surgery seems to be an effective longterm solution for morbidly obese patients and can improve problems of infertility.

\section{Compliance with Ethical Standards}

Conflict of Interest The authors declare that they have no conflict of interest.

Ethics Approval and Consent to Participate Informed consent was obtained from all individual participants included in the study.

\section{References}

1. Narayanan RP, Syed AA. Pregnancy following bariatric surgerymedical complications and management. Obes Surg. 2016

2. Mead NC, Sakkatos P, Sakellaropoulos GC, Adonakis GL, Alexandrides TK, Kalfarentzos F. Pregnancy outcomes and nutritional indices after 3 types of bariatric surgery performed at a single institution. Surg Obes Relat Dis. 2014;10(6):1166-73. 